other countries/cultures. My 2017 scholarship took me to Canada and I visited four cities in two provinces to explore their nursing education and nursing roles. I visited education institutes, care homes, hospitals, nursing agencies and hospices and undertook shadowing a community palliative care team.

As a result of this fantastic experience I have made connections with a hospice in Toronto and we have hosted a visit to our hospice from their care team so we can share learning and experiences. We have also hosted a nurse lecturer from Ottawa who visited us to see how palliative and end of life care is supported in our healthcare system.

This scholarship allowed me to consider the nursing workforce challenges; recruitment, retention and morale. Learning about nursing education from others can help us consider how we support the nursing workforce to transform career opportunities from apprentice to advanced and consultant level nurses in hospice care.

Our hospice is currently developing talent management and a pipeline of nurses:

- Supported care assistants using apprenticeship standards to start their career

- Supporting four Trainee Nursing Associates who are due to graduate in January 2019

- Undertaking an organisational workforce plan, which is supported by us completing functional mapping with teams to identify current and future skills required to deliver services; in turn identify gaps so we can educate, train and upskill to meet gaps

- Working with our Sustainability and Transformation Plan partners across our system to be part of a talent management and workforce planning solution.

This work can be showcased to demonstrate how working in a system and with partners will support workforce development to transform care.

\section{P-282 EVALUATING 'THE ROTATIONAL POST - END OF LIFE CARE, CANCER CARE AND CARE OF THE ELDERLY' FOR BAND 5}

'Brigid Williams, ${ }^{2}$ Paul Newyon. 'Greenwich and Bexley Community Hospice, London, UK; ${ }^{2}$ University of Greenwich, London, UK

\subsection{6/bmjspcare-2018-hospiceabs.307}

Background The National Survey of Bereaved People reported that patients' relatives rated hospital doctors and nurses less well than those working in other settings at ensuring that their loved ones were treated with dignity and respect (Office for National Statistics, 2016). The Nursing and Midwifery Council updated the Code of Conduct for nurses and midwives highlighting the importance of delivering fundamental care to dying people so that they can expect to receive the high standard of care afforded to any other person needing nursing care. The 'Rotational Post' project offered developing Band 5 nurses (within three years of qualifying) the opportunity to work in three care settings (1. Hospice in-patient; 2. Cancer care; 3. Care of the Elderly) over the course of a year (in four month blocks) with support from palliative care professionals, managers and the project lead.

Aims To develop skills and confidence in meeting the needs of people approaching end of life in any care setting. Developing a culture where death is not seen as a failure but whereby an expected death is seen as a successful outcome. Promote greater working partnerships between care providers.

Methods A qualitative study adapting a research case study approach to evaluate the project. Qualitative focus groups (with participating nursing staff) and semi-structured interviews (with stakeholders) were conducted and analysed to assess the perceived impact the project had on stakeholders' and participating nurses' perceptions and experiences, and whether the project had, according to participants' accounts, attained its original aims.

Conclusion Overall the course had a positive impact on the nurses and key stakeholders alike. A number of challenges were experienced throughout the programme including recruitment processes, communication and placements. The evaluation created an opportunity to explore these challenges and how they might be improved in future collaborations of this type.

\section{P-283 DEVELOPMENT OF NURSE PRACTITIONER ROLE IN HOSPICE IN-PATIENT SETTING}

Rosemarie Finnegan, Jo Taylor, Lucy Roth. Teesside Hospice, Middlesbrough, UK

\subsection{6/bmjspcare-2018-hospiceabs.308}

Background Nurse Practitioners have a pivotal role in supporting clinical teams, enhancing patient care by bridging the gap between medical staff, nursing staff and other allied healthcare professionals in many healthcare settings. A clinical need was identified within our hospice team to support enhanced patient care.

Aims Nurse practitioners assess patients and develop appropriate management plans with the medical team and improve continuity of care. They aim to support and develop the skills of less experienced members of nursing staff. They work closely with all members of the multi-disciplinary team facilitating timely interventions and appropriate use of resources. Management and leadership commitments are integral to the role. The Nurse Practitioner, a senior nursing post, encourages career progression within the hospice nursing team. They act as a role model professionally, developing the confidence and competence within the nursing team more broadly.

Method Three nurses with advanced clinical skills were appointed as trainee nurse practitioners in January 2017. After one year, the role was evaluated using a short questionnaire circulated to all clinical and administrative staff. Specific questions were asked about impact on clinical practice, improvement of patient safety, improvement of education and learning needs and communication between team members. Respondents were also given the opportunity to write comments. Post-holders were encouraged to complete a reflective learning log.

Results A 72\% response rate was received from questionnaires. Feedback was positive and informative. A thematic analysis was undertaken. The five identified themes have allowed us to develop the role and its integration within the clinical team. The post-holders have used reflection to identify individual learning needs.

Conclusion Due to the success of this innovative role, the organisation has continued to support the nurse practitioner role and also appointed two 'out reach' nurse practitioners to support patient care in the community. 